\title{
Nonstandard Absorption on Donors in Uniformly Doped II-VI 0D Structures
}

\author{
K. KArPIERZ ${ }^{a}$, M. SzOT ${ }^{a, b}$ And M. GRYNBERG ${ }^{a}$ \\ ${ }^{a}$ Institute of Experimental Physics, Warsaw University \\ Hoża 69, 00-681 Warsaw, Poland \\ ${ }^{b}$ Institute of Physics, Polish Academy of Sciences \\ al. Lotników 32/46, 02-668 Warsaw, Poland
}

\begin{abstract}
The new approach to the understanding of intrashallow donor transition in the reduced dimensionality systems is presented. The magnetospectroscopy experiments done on the $\mathrm{CdTe} / \mathrm{CdMgTe}$ quantum well based samples, uniformly $n$-doped, show indications that the surprising lack of spectral sensitivity on applied photon energy can be understood as a result of sample response coming from its different regions. This "non spectroscopic" behaviour (in a sense of the Zeeman splitting) is a consequence of the properties of systems with reduced dimensionality where variety of centre locations in the structure results in continuous density of states available for absorption.
\end{abstract}

PACS numbers: 71.55.Gs, 72.15.Rn, 72.80.Ey, 73.21.Fg

\section{Introduction}

The absorption processes which are considered in spectroscopy experiments always rely on single centre/centres which may absorb incident photon(s). The example of such spectroscopy is magnetospectroscopy of shallow donors [1], where one splits energy levels of a single centre by applying the external magnetic field. As a result, for different magnetic fields, the different photon energies are absorbed. This spectroscopy gives conclusions about the whole energy level structure and its dependence on magnetic field. For the 3D samples each centre in the structure is surrounded by the uniform vicinity of the host lattice and therefore one can consider every centre as identical with all others. The sample spectral response is reflecting the response of single centre. The quite another situation occurs in the reduced dimensionality systems like quantum well $(\mathrm{QW})$, where the structure of energy levels for each shallow donor centre depends on its location in the structure. In the 2D structures, for Coulombic centres, in the extreme cases one can expect the energy difference even of the factor 16 between the donors in the QW centre 
and at the QW interface [2]. For the particular case of our sample the energy of the basic $1 s-2 p^{+}$transition is varying from $\approx 2 \mathrm{meV}$ (for donors in the barrier) up to $\approx 16 \mathrm{meV}$ (for donors in the middle of QW). In such situation, for given experimental conditions (incident photon energy, magnetic field strength), the sample response is originating only from a certain part of all centres in the sample - from these, which match the energy splitting with the photon energy. By varying the experimental conditions one may get the response from quite different regions of the sample. The same happens for centres in all surroundings with the reduced dimensionality. The example and identification of such a case for the $0 \mathrm{D}$ environment is presented below. The goal of this paper is to present the explanation and conclusions coming from donors surrounded by such environment.

\section{Samples and experiment}

Samples were based on $\mathrm{CdTe} / \mathrm{Cd}_{0.8} \mathrm{Mg}_{0.2} \mathrm{Te}$ single $\mathrm{QW}, 16 \mathrm{~nm}$ wide and $240 \mathrm{meV}$ deep in the conduction band with the barriers width $48 \mathrm{~nm}$ and $480 \mathrm{~nm}$, respectively. The whole structure (the well and the barriers) was uniformly bulk doped with iodine at the level of $\approx 2 \times 10^{16} \mathrm{~cm}^{-3}$. The measurements at the temperature of $4.2 \mathrm{~K}$ of photocurrent due to far infrared (FIR) illumination by monochromatic light with the photon energies from $6.6 \mathrm{meV}$ up to $17.6 \mathrm{meV}$ as a function of external magnetic field have been carried out. The photocurrent due to chopped FIR illumination was measured by lock-in technique. For that kind of samples one expects the strong local spatial changes of electric potential potential fluctuations [3]. The sources of them are noncompensated surface states, ionized donor states, and charged states which accompany the dislocations. It is worth noticing that they influence the shallow donor states even when the source of potential is located relatively far from them. Therefore the whole density of all "active" fluctuation sources is much bigger than in other systems. As a result of potential fluctuations one gets the additional confinement of electrons in the quantum well. The confinement along the $O Z$ (growth) direction is due to the QW band structure and the confinement in $O X Y$ plane is introduced by the local potential fluctuations. We can consider the carriers and local centres as "embedded" in the 0D surroundings — a kind of quantum dot (QD). Since the confinement is done partially by the "structure engineering" (growth process of QW) and partially by the influence of intrinsic electric fields - that kind of QD might be called mixed quantum dot - MQD [4]. For our samples the density of the MQDs does not vary much from the donor density. One can estimate that each single MQD contains at most a few donor centres.

The second point, one has to remember, is that in the sample the uniform doping gives the condition of different spatial locations of donor centres with respects to QW interface and MQD midpoint. This, in turn, results in different $1 s-2 p^{ \pm 1}$ intradonor transition energies $[1,2]$. It means that in the two neighbouring MQDs there might be embedded two donors of completely different binding 
energies. In order to perform spectroscopy of these donors one has to apply different conditions of experiment and then one gets the spectral response from two different places (MQDs) in the sample. Additionally, for these samples in the state of thermodynamic equilibrium, at low temperatures, all donors centres are not populated - electrons are localized onto the compensation centres in the barriers and/or CdTe/substrate interface [3]. The illumination with the light of over band gap photon energy leads to the generation of excess free carriers. They conduct current, causing the drop of sample resistance to the level enabling the photocurrent experiments. In our experiments the constant illumination with the photon energy $h \nu=2.08 \mathrm{eV}$ - over band gap for the well and for the barrier, was used.

\section{Results}

Figure 1 shows the results of experiments done with illumination of the sample with different FIR photons. One observes, except the traces of GaAs substrate $3 \mathrm{D}$ shallow donor response, the clear maximum of the signal, which occurs for all spectra for magnetic field $\approx 2.2 \mathrm{~T}$. By changing the photon energy by a factor of almost 3 one would expect the shift of the line if it were the spectral one - originating from the same donor centre. This is not the case of these
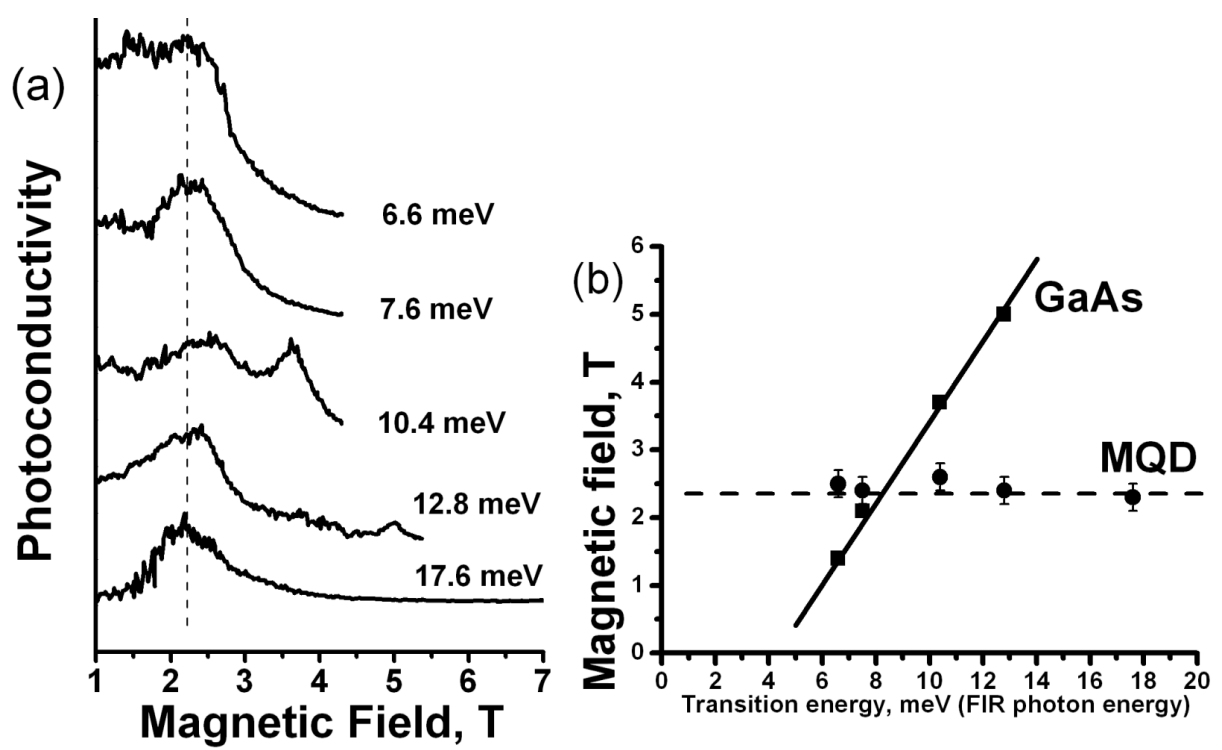

Fig. 1. (a) Photoconductivity spectra due to FIR illumination, taken at $T=4.2 \mathrm{~K}$. Each spectrum is marked by the value of FIR photon energy. The spectra were shifted for clarity. The dashed line shows the maxima of peaks due to magnetic field enhanced localization. Part (b) shows the magnetic field maxima for peaks due to intradonor transition in GaAs substrate and due to localization in CdTe/CdMgTe structure with MQD. 
results. In order to understand all spectra one has to show why the magnetic field induces the enhanced photoconductivity (PC) signal for that value of magnetic field. Such mechanism has been proposed in [5]. It is based on the fact that the external magnetic field modifies the wave functions of carriers known in the literature as cyclotron resonance. For certain magnetic field $B^{*}$ the corresponding cyclotron orbit radius $R_{\text {cyclotron }}$ fits the characteristic spatial dimensions $R_{\mathrm{MQD}}$ of the existing in the structure MQDs. For increasing $B$, and thus decreasing carrier cyclotron radius, one expects the enhancement of electron capture probability onto the centres within the MQD extent for the external magnetic fields greater than $B^{*}$. It is shown schematically in Fig. 2. This gives the increase in PC signal starting from $\approx B^{*}$. In our case this enables the spectroscopy of intrashallow donor transitions, which can be, in principal, monitored for the fields greater than $B^{*}$.

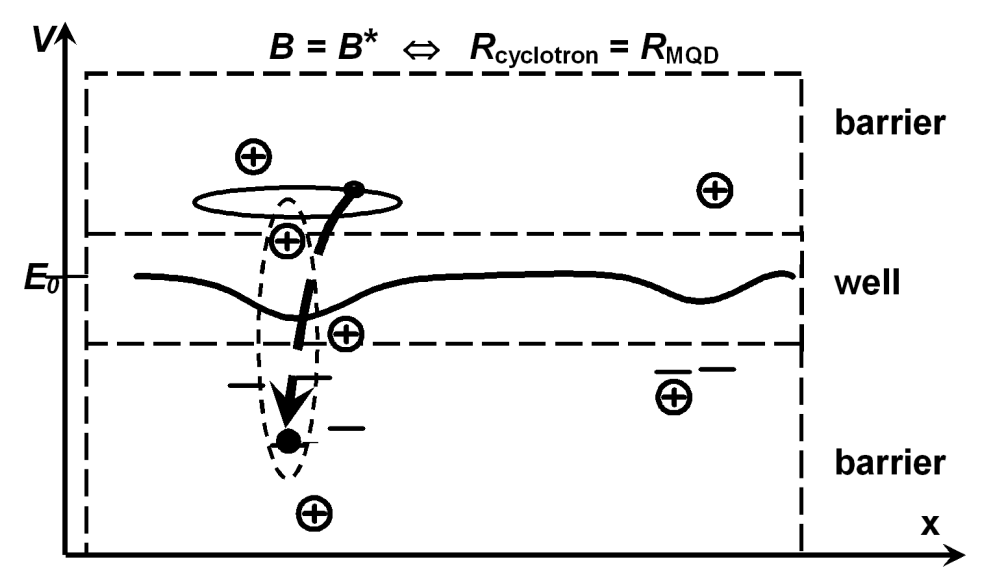

Fig. 2. Scheme of magnetic field induced localization of electrons in the conduction band onto the centres within the spatial extent of MQD. Symbol $\bigoplus$ represents the location of ionized donor in the structure. The appropriate energy levels are marked by the horizontal short lines. $E_{0}$ - energy of the bottom of the first electric level in the quantum well. The solid curved line - the relief of the bottom of conduction band due to the MQW existence.

Starting from that point the understanding of the processes in MQDs system is quite different from the classical donor spectroscopy, like in 3D surroundings. In our system, due to uniform doping, one deals with the whole spectrum of shallow donor binding energies resulting in the existence of the quasi continuum of available $1 s-2 p^{ \pm}$transitions in our sample. Therefore, the magnetospectroscopy of shallow donors, taken at fixed transition energy - fixed incident photon energy, exhibits unexpected features. Each single FIR photon "chooses" the appropriate donor centre. The condition of "choice" is that the donor splitting energy at a given magnetic field fits to the photon energy. For a given incident photon energy, while changing the magnetic field, one leads to the field induced localization onto all 
shallow donors and then to the interplay between different donors. Only a part of them does give a contribution to the PC signal. For photons of another energy the same interplay leads to enhancing the PC signal which is governed by the same magnetic field induced localization, giving in our case, the signal rise at $\approx 2.2 \mathrm{~T}$. The spectral response of the whole sample is a result of absorption occurring on the certain group of donors only - different for different FIR photon energies. Figure 1 shows that one observes the same maximum of PC signal, regardless the incident photon energy. This is apparently surprising but one can understand it assuming that donors differently located in the QW and MQD are responsible for each spectra.

\section{Conclusions}

We point out that the described mechanism of getting the PC signal maxima at the same magnetic field for different photon energies is not quite similar to the classical understanding of shallow donor spectroscopy. By applying the illumination of different FIR photon energies one gets the signal from donors located in another way in the structure. Secondly: the "driving force" of the possibility of performing spectroscopy is the application of the external magnetic field. This field drives the enhanced confinement of electron which gets localized onto the empty centres when cyclotron radius approaches the size of quantum dot.

\section{References}

[1] G. Bastard, Phys. Rev. B 24, 4714 (1981).

[2] R.L. Green, K.K. Bajaj, Phys. Rev. B 34, 951 (1986).

[3] M. Szot, K. Karpierz, J. Kossut, M. Grynberg, in: 27th Int. Proc. Conf. Phys. of Semicond. ICPS-27, Eds. J. Menéndez, C.G. Van de Valle, AIP Conf. Proc., Vol. 772, AIP, New York 2005, p. 1129.

[4] K. Karpierz, J. Łusakowski, M. Szot, J. Kossut, M. Grynberg, J. Phys. C, to be published.

[5] M. Szot, K. Karpierz, J. Kossut, M. Grynberg, in: 28th Int. Proc. Conf. Phys. of Semicond. ICPS-28, Eds. W. Jantsch, F. Schäfller, AIP Conf. Proc., Vol. 893, Springer, Berlin 2007, p. 251. 